\title{
The $G^{\prime} / G^{2}$ - Expansion Method for Solving Fractional Burgers - Fisher and Burgers Equations
}

\author{
Abaker A. Hassaballa ${ }^{1,2}$ \\ ${ }^{1}$ Department of Mathematics, College of Applied \& Industrial Sciences, Bahri University, Khartoum, Sudan \\ ${ }^{2}$ Department of Mathematics, Faculty of Science, Northern Border University, Arar, KSA \\ Email address: \\ Abakerh@gmail.com \\ To cite this article: \\ Abaker A. Hassaballa. The $G^{\prime} / G^{2}$ - Expansion Method for Solving Fractional Burgers -Fisher and Burgers Equations. Applied and \\ Computational Mathematics. Vol. 9, No. 3, 2020, pp. 56-63. doi: 10.11648/j.acm.20200903.12 \\ Received: April 18, 2020; Accepted: May 12, 2020; Published: May 27, 2020

\begin{abstract}
In this paper, we apply $G^{\prime} / G^{2}$-Expansion method to discover a strategy for the approximate solution of the generalized fractional Burger-Fisher equation and fractional Burger equation. The given fractional Burger-Fisher and burger equation through substitution are converted into nonlinear ordinary differential equations, in the sense of the Jumarie's modified Riemann-Liouville fractional derivative. The travelling wave solution is approximated by the $G^{\prime} / G^{2}$-Expansion method with unknown parameters that can be expressed by trigonometric functions, exponential functions, hyperbolic functions and rational functions. These results reveal that the proposed method is very effective and simple in performing a solution to the nonlinear fractional partial differential equation.
\end{abstract}

Keywords: $G^{\prime} / G^{2}$-expansion Method, Burgers-Fisher Equation, Burgers Equation

\section{Introduction}

The fractional partial differential equations have a wide range of applications in several branch of the pure and applied sciences. They appear for instance in various applications in physics, biology, engineering and many others. The generalized fractional Burgers-Fisher equation is of eminent importance for describing different mechanisms. Burgers-Fisher equation has important applications in various areas of financial mathematics, gas dynamic, traffic flow, number theory, heat conduction, elasticity, applied mathematics and physics applications. Burgers-Fisher equation is a highly nonlinear equation because it is a combination of reaction, convection and diffusion mechanisms, this equality is called Burgers-Fisher because it possesses the properties of convective phenomenon from Burgers equation and having diffusion transport as well as reaction kind of characteristics from Fisher equation.

Many Researchers have used various numerical for obtaining exact solution of nonlinear fractional partial differential equations. For instances, fractional power series solutions method [1, 2, 3, 7], reproducing kernel method [4], simplest equation method [5], fractional mapping expansion method [6], $\left(G^{\prime} / G\right)$-expansion method [8], first integral method [9], conformable fractional derivative [10, 11, 12, 16], $\left(G^{\prime} / G^{2}\right)$ expansion method [13], kernel Hilbert space method [14], efficient analytical method [18], generalized Kudryashov method [15].

The paper is structured follows: Section 1 gives an introduction of the study. Section 2 gives definition of fractional calculus. In Section 3, we provide the description of the method to be used in the paper. Section 4 presents the application schemes of the afore-mentioned method, explaining the techniques to be used in the analysis. Finally, the manuscript ended up with the conclusion in Section 5.

\section{Fractional Calculus}

In this paper, before embarking into detail of an $\left(G^{\prime} / G^{2}\right)$ expansion method, we would like to recall some basic definitions, results, and properties of the fractional calculus operators, used in the remaining part of the article.

Definition 1.

The Jumarie's modified Riemann- Liouville fractional derivative of order $\alpha$ is defined as follows (Jumarie's, 
2006).

$$
D^{\alpha} f(t)= \begin{cases}\frac{1}{\Gamma(-\alpha)} \frac{d}{d t} \int_{0}^{t}(t-\tau)^{-\alpha-1}(f(\tau)-f(0)) d \tau, & \alpha<0, \\ \frac{1}{\Gamma(1-\alpha)} \frac{d}{d t} \int_{0}^{t}(t-\tau)^{-\alpha}(f(\tau)-f(0)) d \tau, & 0<\alpha<1, \\ {\left[f^{(\alpha-n)}(t)\right]^{n},} & \mathrm{n} \leq \alpha<\mathrm{n}+1, \mathrm{n} \geq 1,\end{cases}
$$

If $\alpha=0$, then $D_{a}^{\alpha} f(t)=f(t)$, where $\Gamma(\alpha)=\int_{0}^{\infty} t^{\alpha-1} e^{-t} d t$

is gamma function.

Some useful results of Jumarie's modified RiemannLiouville fractional derivative as follows:

$$
\begin{gathered}
D_{t}^{\alpha} t^{\beta}=\frac{\Gamma(1+\beta)}{\Gamma(1+\beta-\alpha)} t^{\beta-\alpha} \\
D_{t}^{\alpha}(c f(t))=c D_{t}^{\alpha}(f(t)), c \text { is constant, } \\
D_{t}^{\alpha}[a f(t)+b g(t)]=a D_{t}^{\alpha} f(t)+b D_{t}^{\alpha} g(t), \\
P\left(u_{1} \ldots u_{k}, \frac{\partial u_{1}}{\partial t} \ldots \frac{\partial u_{k}}{\partial t}, \frac{\partial u_{1}}{\partial x_{1}} \ldots \frac{\partial u_{k}}{\partial x_{1}}, \ldots, \frac{\partial u_{1}}{\partial x_{n}} \ldots \frac{\partial u_{k}}{\partial x_{n}}, D_{t}^{\alpha} u_{1} \ldots, D_{t}^{\alpha}\right. \\
\text { Where } u=u\left(t, x_{1}, x_{2}, \ldots, x_{n}\right) \text { and } P \text { is polynomial of } u, \text { the } \\
\text { travelling wave solutions of Eq. (6) by use transformation, } \\
u\left(t, x_{1}, x_{2}, \ldots, x_{n}\right)=U(\zeta), \zeta=\zeta\left(t, x_{1}, x_{2}, \ldots, x_{n}\right)
\end{gathered}
$$$$
P\left(u_{1} \ldots u_{k}, \frac{\partial u_{1}}{\partial t} \ldots \frac{\partial u_{k}}{\partial t}, \frac{\partial u_{1}}{\partial x_{1}} \ldots \frac{\partial u_{k}}{\partial x_{1}}, \ldots, \frac{\partial u_{1}}{\partial x_{n}} \ldots \frac{\partial u_{k}}{\partial x_{n}}, D_{t}^{\alpha} u_{1} \ldots, D_{t}^{\alpha} u_{k}, D_{x_{1}}^{\beta} u_{1} \ldots, D_{x_{1}}^{\beta} u_{k}, \cdots, D_{x_{n}}^{\beta} u_{1} \ldots, D_{x_{n}}^{\beta} u_{k}, \ldots\right)=0, \quad 0<\alpha<\beta<1
$$

where $a$ and $b$ are constant, and $D_{t}^{\alpha} a=0$

$$
D_{t}^{\alpha}(f(t) g(t))=g(t) D_{t}^{\alpha} f(t)+f(t) D_{t}^{\alpha} g(t)
$$

\section{Description of the $G^{\prime} / G^{2}$ - Expansion Method for Fractional}

In this section, we give the main steps of the $\left(G^{\prime} / G^{2}\right)-$ expansion method for solving nonlinear fractional partial differential equations (NFPDEs) as follow:

i. We consider the nonlinear fractional partial differential equation as,
Convert the partial differential Eq. (6) into the following nonlinear ordinary differential equation,

$$
Q\left(U_{1} \ldots, U_{k}, U_{1}^{\prime} \ldots U_{k}^{\prime}, U_{1}^{\prime \prime} \ldots, U_{k}^{\prime \prime}, U_{1}^{\prime \prime \prime} \ldots U_{k}^{\prime \prime \prime}, \cdots\right)=0
$$

Where $Q$ is polynomial of $U(\zeta)$ and its various derivatives the prime denotes the derivation with respect to $\zeta$.

ii. We suppose that the solution of Eq. (8) can be expressed by a polynomial in $\left(G^{\prime} / G^{2}\right)$ such that:

$$
U(\zeta)=a_{0}+\sum_{i=1}^{m}\left[a_{i}\left(\frac{G^{\prime}}{G^{2}}\right)^{i}+b_{i}\left(\frac{G^{\prime}}{G^{2}}\right)^{-i}\right]
$$

Where $a_{0}, a_{i}$ and $b_{i},(i=1,2,3, \ldots m)$ are constants to be determined, with $a_{m}$ or $b_{m}$ may be zero, but both of them cannot be zero, the value of $m$ is a positive integer which will be determined, the parameter $m$ is usual higher by balancing the highest order derivatives with the nonlinear terms in Eq. (8).

Where $G=G(\zeta)$ satisfies a second order linear ordinary differential equation in the form

$$
\left(\frac{G^{\prime}}{G^{2}}\right)^{\prime}=\mu+\lambda\left(\frac{G^{\prime}}{G^{2}}\right)^{2}
$$

Where $\mu \neq 1$ and $\lambda \neq 0$ are integers.

Substituting Eq. (9) using Eq. (10) into Eq. (8). Then collecting all terms with same order of $\left(G^{\prime} / G^{2}\right)$ together. Then equating each coefficient of the resulting polynomial to zero, we obtain a set of algebraic equations for parameters $a_{0}, a_{i}, \mathrm{~b}_{i}, \lambda$ and $\mu$.

iii. Having the values $a_{0}, a_{i}, \mathrm{~b}_{i}, \lambda$ and $\mu$ from (ii) and the solution of Eq. (8), which can be obtained, we are closed to the solution of the nonlinear evolution Eq. (6).

The general solutions can be categorized into the following three cases when A and B are arbitrary nonzero constants.

If $\lambda \mu>0$, then we obtain the general solution

$$
\frac{G^{\prime}(\zeta)}{G^{2}(\zeta)}=\sqrt{\frac{\lambda}{\mu}}\left[\frac{A \cos (\sqrt{\lambda \mu} \zeta)+B \sin (\sqrt{\lambda \mu} \zeta)}{B \cos (\sqrt{\lambda \mu} \zeta)-A \sin (\sqrt{\lambda \mu} \zeta)}\right]
$$

If $\lambda \mu<0$, then we obtain the general solution 


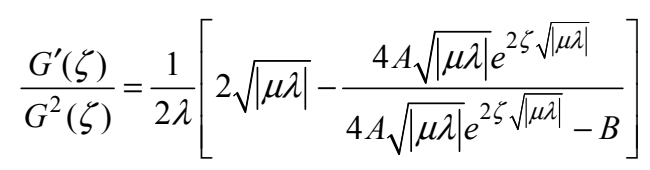

Which is equivalent to

$\frac{G^{\prime}(\zeta)}{G^{2}(\zeta)}=-\frac{\sqrt{|\lambda \mu|}}{\lambda}\left[\frac{A \cosh (2 \sqrt{|\lambda \mu|} \zeta)+A \sinh (2 \sqrt{|\lambda \mu|} \zeta)+B}{A \cosh (2 \sqrt{|\lambda \mu|} \zeta)+A \sinh (2 \sqrt{|\lambda \mu|} \zeta)-B}\right]$

If $\lambda \neq 0, \mu=0$, then we obtain the general solution

$$
\frac{G^{\prime}(\zeta)}{G^{2}(\zeta)}=-\frac{A}{\lambda(B+A \zeta)}
$$

\section{Applications of the $G^{\prime} / G^{2}$ - Expansion Method}

In this section, we use the $\left(G^{\prime} / G^{2}\right)$-expansion Method on time fractional Burger-Fisher equation and time fractional Burger equation.

Example (1)

We consider the time fractional Burger-Fisher equation

$$
\frac{\partial^{\alpha} u}{\partial t^{\alpha}}+\rho u \frac{\partial u}{\partial x}-\frac{\partial^{2} u}{\partial x^{2}}=\beta u(1-u)
$$

where $\rho$ and $\beta$ are parameters and $0<\alpha<1$

Will be converted the time fractional transform given by

$$
u(x, t)=U(\zeta), \zeta=\mathrm{kx}+\frac{\sigma t^{\alpha}}{\Gamma(1+\alpha)}
$$

Where $k$ and $\sigma$ are constants. Substituting Eq. (16) into Eq. (15) we obtain the following ordinary differential equation

$$
\sigma U^{\prime}+\rho k U U^{\prime}-k^{2} U^{\prime \prime}-\beta U+\beta U^{2}=0
$$

With $U^{\prime}=\frac{d U}{d \zeta}$. Balance the nonlinear term $u^{2}$ with the highest order derivative $U^{\prime \prime}$ gives $m=2$, that does not lead to any solution, however, balancing $U U^{\prime}$ with $U^{\prime \prime}$ gives $m=1$,

$$
U(\zeta)=a_{0}+a_{1}\left(\frac{G^{\prime}}{G^{2}}\right)+b_{1}\left(\frac{G^{\prime}}{G^{2}}\right)^{-1}
$$

Where $a_{1}, b_{1}$ and $a_{0}$ arbitrary constants.

Substituting Eq. (18) with Eq. (10) into Eq. (17), then collecting all terms with the same power of $\left(G^{\prime} / G^{2}\right)$ and setting each coefficient of power of $\left(G^{\prime} / G^{2}\right)$ to zero, we achieve a system of algebraic equations and solving these algebraic equations gives sets of solutions.

Case 1:

$$
k=0, \mu=\mu, \lambda=-\frac{\beta^{2}}{4 \sigma^{2} \mu}, \sigma=\sigma, a_{0}=\frac{1}{2}, a_{1}=0, b_{1}=\frac{\sigma \mu}{\beta},
$$

Case 2:

$$
k=0, \mu=\frac{\beta}{16 \sigma a_{1}}, \lambda=-\frac{\beta a_{1}}{\sigma}, \sigma=\sigma, a_{0}=\frac{1}{2}, a_{1}=a_{1}, b_{1}=\frac{1}{16 a_{1}},
$$

Case 3:

$$
k=0, \mu=-\frac{\beta^{2}}{4 \sigma^{2} \lambda}, \lambda=\lambda, \sigma=\sigma, a_{0}=\frac{1}{2}, a_{1}=-\frac{\sigma \lambda}{\beta}, b_{1}=0
$$

Case 4:

$$
k=k, \mu=-\frac{\rho^{2}}{16 k^{2} \lambda}, \lambda=\lambda, \sigma=-\frac{k\left(\rho^{2}+4 \beta\right)}{2 \rho}, a_{0}=\frac{1}{2}, a_{1}=\frac{2 k \lambda}{\rho}, b_{1}=0
$$

Case 5:

$$
k=k, \mu=\mu, \lambda=-\frac{\rho^{2}}{16 k^{2} \mu}, \sigma=-\frac{k\left(\rho^{2}+4 \beta\right)}{2 \rho}, a_{0}=\frac{1}{2}, a_{1}=0, b_{1}=-\frac{2 k \mu}{\rho}
$$

Case 6: 


$$
k=-\frac{\rho}{32 a_{1} \mu}, \mu=\mu, \lambda=-16 a_{1}^{2} \mu, \sigma=-\frac{\rho^{2}+4 \beta}{64 a_{1} \mu}, a_{0}=\frac{1}{2}, a_{1}=a_{1}, b_{1}=\frac{1}{16 a_{1}},
$$

Substituting Eq. (19) with Eq. (13) into Eq. (18) and simplify, we obtain the following.

$$
U_{1}(x, t)=\frac{1}{2}+\frac{1}{2}\left(\frac{A \cosh \left(\frac{\beta t^{\alpha}}{\Gamma(1+\alpha)}\right)+A \sinh \left(\frac{\beta t^{\alpha}}{\Gamma(1+\alpha)}\right)+B}{A \cosh \left(\frac{\beta t^{\alpha}}{\Gamma(1+\alpha)}\right)+A \sinh \left(\frac{\beta t^{\alpha}}{\Gamma(1+\alpha)}\right)-B}\right)^{-1}
$$

Substituting Eq. (20) with Eq. (13) into Eq. (18) and simplify, we obtain the following.

$$
\begin{aligned}
U_{2}(x, t)= & \frac{1}{2}+\frac{1}{4}\left(\frac{A \cosh \left(\frac{\beta t^{\alpha}}{2 \Gamma(1+\alpha)}\right)+A \sinh \left(\frac{\beta t^{\alpha}}{2 \Gamma(1+\alpha)}\right)+B}{A \cosh \left(\frac{\beta t^{\alpha}}{2 \Gamma(1+\alpha)}\right)+A \sinh \left(\frac{\beta t^{\alpha}}{2 \Gamma(1+\alpha)}\right)-B}\right) \\
& +\frac{1}{4}\left(\frac{A \cosh \left(\frac{\beta t^{\alpha}}{2 \Gamma(1+\alpha)}\right)+A \sinh \left(\frac{\beta t^{\alpha}}{2 \Gamma(1+\alpha)}\right)+B}{A \cosh \left(\frac{\beta t^{\alpha}}{2 \Gamma(1+\alpha)}\right)+A \sinh \left(\frac{\beta t^{\alpha}}{2 \Gamma(1+\alpha)}\right)-B}\right)^{-1}
\end{aligned}
$$

Substituting Eq. (21) with Eq.(13) into Eq. (18) and simplify, we obtain the following.

$$
U_{3}(x, t)=\frac{1}{2}-\frac{1}{2}\left(\frac{A \cosh \left(\frac{\beta t^{\alpha}}{\Gamma(1+\alpha)}\right)+A \sinh \left(\frac{\beta t^{\alpha}}{\Gamma(1+\alpha)}\right)+B}{A \cosh \left(\frac{\beta t^{\alpha}}{\Gamma(1+\alpha)}\right)+A \sinh \left(\frac{\beta t^{\alpha}}{\Gamma(1+\alpha)}\right)-B}\right)
$$

Substituting Eq. (22) with Eq. (13) into Eq. (18) and simplify, we obtain the following

$$
U_{4}(x, t)=\frac{1}{2}-\frac{1}{2}\left(\frac{A \cosh \left(\frac{\rho_{\mathrm{x}}}{2}-\frac{\left(\rho^{2}+4 \beta\right) t^{\alpha}}{4 \Gamma(1+\alpha)}\right)+A \sinh \left(\frac{\rho \mathrm{x}}{2}-\frac{\left(\rho^{2}+4 \beta\right) t^{\alpha}}{4 \Gamma(1+\alpha)}\right)+B}{A \cosh \left(\frac{\rho \mathrm{x}}{2}-\frac{\left(\rho^{2}+4 \beta\right) t^{\alpha}}{4 \Gamma(1+\alpha)}\right)+A \sinh \left(\frac{\rho \mathrm{x}}{2}-\frac{\left(\rho^{2}+4 \beta\right) t^{\alpha}}{4 \Gamma(1+\alpha)}\right)-B}\right)
$$

Substituting Eq. (23) with Eq. (13) into Eq. (18) and simplify, we obtain the following.

$$
U_{5}(x, t)=\frac{1}{2}-\frac{1}{2}\left(\frac{A \cosh \left(\frac{\rho x}{2}-\frac{\left(\rho^{2}+4 \beta\right) t^{\alpha}}{4 \Gamma(1+\alpha)}\right)+A \sinh \left(\frac{\rho x}{2}-\frac{\left(\rho^{2}+4 \beta\right) t^{\alpha}}{4 \Gamma(1+\alpha)}\right)+B}{A \cosh \left(\frac{\rho x}{2}-\frac{\left(\rho^{2}+4 \beta\right) t^{\alpha}}{4 \Gamma(1+\alpha)}\right)+A \sinh \left(\frac{\rho x}{2}-\frac{\left(\rho^{2}+4 \beta\right) t^{\alpha}}{4 \Gamma(1+\alpha)}\right)-B}\right)^{-1}
$$

Substituting Eq. (24) with Eq. (13) into Eq. (18) and simplify, we obtain the following. 


$$
\begin{aligned}
U_{6}(x, t)=\frac{1}{2}+\frac{1}{4}\left(\frac{A \cosh \left(\frac{\rho \mathrm{x}}{4}+\frac{\left(\rho^{2}+4 \beta\right) t^{\alpha}}{8 \Gamma(1+\alpha)}\right)-A \sinh \left(\frac{\rho \mathrm{x}}{4}+\frac{\left(\rho^{2}+4 \beta\right) t^{\alpha}}{8 \Gamma(1+\alpha)}\right)+B}{A \cosh \left(\frac{\rho \mathrm{x}}{4}+\frac{\left(\rho^{2}+4 \beta\right) t^{\alpha}}{8 \Gamma(1+\alpha)}\right)-A \sinh \left(\frac{\rho \mathrm{x}}{4}+\frac{\left(\rho^{2}+4 \beta\right) t^{\alpha}}{8 \Gamma(1+\alpha)}\right)-B}\right) \\
+\frac{1}{4}\left(\frac{\left.A \cosh \left(\frac{\rho \mathrm{x}}{4}+\frac{\left(\rho^{2}+4 \beta\right) t^{\alpha}}{8 \Gamma(1+\alpha)}\right)-A \sinh \left(\frac{\rho \mathrm{x}}{4}+\frac{\left(\rho^{2}+4 \beta\right) t^{\alpha}}{8 \Gamma(1+\alpha)}\right)+B\right)^{-1}}{A \cosh \left(\frac{\rho \mathrm{x}}{4}+\frac{\left(\rho^{2}+4 \beta\right) t^{\alpha}}{8 \Gamma(1+\alpha)}\right)-A \sinh \left(\frac{\rho \mathrm{x}}{4}+\frac{\left(\rho^{2}+4 \beta\right) t^{\alpha}}{8 \Gamma(1+\alpha)}\right)-B}\right)
\end{aligned}
$$

Graphical representation of the solutions of Burgers equation:

Solution $U_{1}(x, y)$ is kink waves are traveling waves which arise from one asymptotic state to another. This is presented in Figure 1 to show the shape of the exact solution of fractional Burger-Fisher equation.

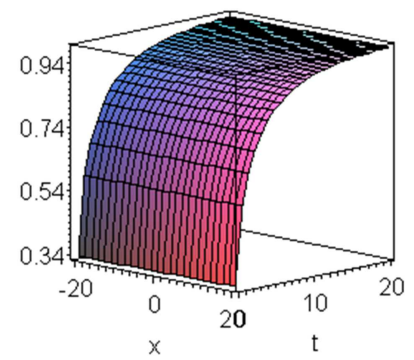

Figure 1. Exact solution for $\beta=1, \mathrm{~A}=3, \mathrm{~B}=6, \alpha=0.5$ with $0 \leq t \leq 20$, $-20 \leq x \leq 20$.

Solution $U_{2}(x, y)$ is singular kink periodic traveling wave solution. This is presented in Figure 2 to show the shape of the exact periodic traveling wave solution of fractional Burger-Fisher equation.

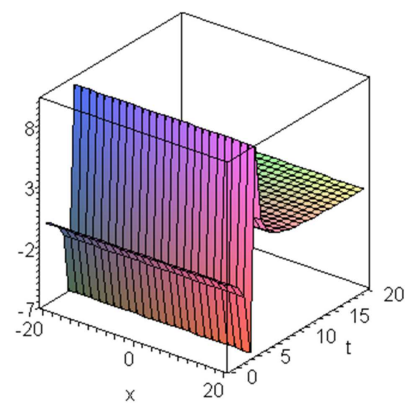

Figure 2. Exact solution for $\beta=\frac{1}{2}, \mathrm{~A}=3, \mathrm{~B}=6, \alpha=0.5$ with $0 \leq t \leq 20,-20 \leq x \leq 20$.

Solution $U_{3}(x, y)$ is singular kink periodic traveling wave solution. This is presented in Figure 3 to show the shape of the exact solution of fractional Burger-Fisher equation.

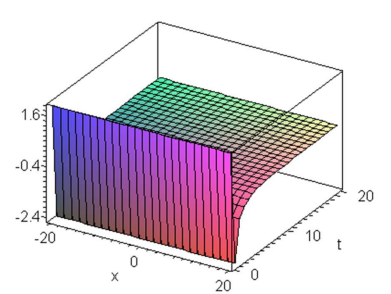

Figure 3. Exact solution for $\beta=1, \mathrm{~A}=3, \mathrm{~B}=9, \alpha=0.5$ with $0 \leq t \leq 20,-20 \leq x \leq 20$.

Presented Soliton periodic traveling wave solution $U_{4}(x, y)$ is singular kink wave solution given in Figure 4 . This figure gives the shape of the exact solution of fractional Burger-Fisher equation.

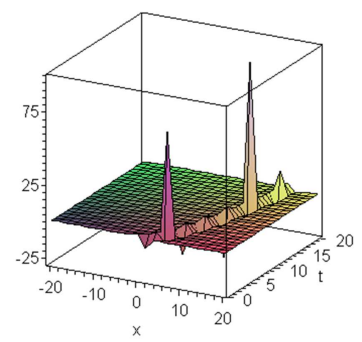

Figure 4. Exact solution for $\beta=1, \rho=1, \mathrm{~A}=3, \mathrm{~B}=6, \alpha=0.5$ with $0 \leq t \leq 20,-20 \leq x \leq 20$.

Solution $U_{5}(x, y)$ is kink waves are traveling waves which arise from one asymptotic state to another. This is presented in Figure 5 to show the shape of the exact solution of fractional Burger-Fisher equation.

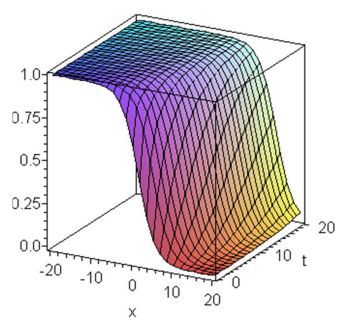

Figure 5. Exact solution for $\beta=1, \rho=1, \mathrm{~A}=3, \mathrm{~B}=6, \alpha=0.75$ with $0 \leq t \leq 20$, $-20 \leq x \leq 20$ 
Presented Soliton periodic traveling wave solution $U_{6}(x, y)$ is singular kink wave solution given in Figure 6 . This figure gives the shape of the exact solution of fractional Burger-Fisher equation.

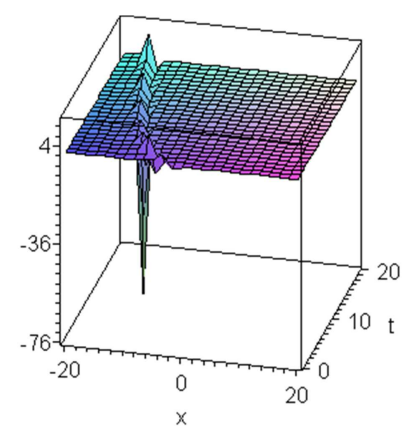

Figure 6. Exact solution for $\rho=1, \beta=1, \mathrm{~A}=3, \mathrm{~B}=6, \alpha=0.5$ with $0 \leq t \leq 20,-20 \leq x \leq 20$.

Example: (2)

We consider the fractional Burger equation

$$
\frac{\partial^{\alpha} u}{\partial t^{\alpha}}+\rho u \frac{\partial^{\alpha} u}{\partial x^{\alpha}}-\delta \frac{\partial^{2 \alpha} u}{\partial x^{2 \alpha}}=0, t>0,0<\alpha<1
$$

Will be converted the fractional complex transform given by

$$
u(x, t)=U(\zeta), \zeta=\frac{k x^{\alpha}}{\Gamma(1+\alpha)}+\frac{\sigma t^{\alpha}}{\Gamma(1+\alpha)}
$$

Where $k$ and $\gamma$ nonzero constants. Substituting Eq. (32) into Eq. (31) we obtain the following ordinary differential equation

$$
\sigma U^{\prime}+\rho k U U^{\prime}-\delta k^{2} U^{\prime \prime}=0
$$

With $U^{\prime}=\frac{d U}{d \zeta}$. By once integrating Eq. (33) with respect to $\zeta$ and setting the constant of integration to zero, we obtain.

$$
\sigma U+\frac{1}{2} \rho k U^{2}-\delta k^{2} U^{\prime}=0
$$

Balance the nonlinear term $U^{2}$ with the highest order derivative $U^{\prime}$ gives $m=1$, then.

$$
U(\zeta)=a_{0}+a_{1}\left(\frac{G^{\prime}}{G^{2}}\right)+b_{1}\left(\frac{G^{\prime}}{G^{2}}\right)^{-1}
$$

Where $a_{1}, b_{1}$ and $a_{0}$ arbitrary constants.

Substituting Eq. (35) with Eq. (10) into Eq. (34), then collecting all terms with the same power of $\left(G^{\prime} / \mathrm{G}^{2}\right)$ together, and Setting each coefficient of power of $\left(G^{\prime} / \mathrm{G}^{2}\right)$ to zero, we achieve a system of algebraic equations and solving these algebraic equations gives sets of solutions

Case 1:

$$
\mu=\mu, \lambda=-\frac{\sigma^{2}}{4 \mu \delta^{2} k^{4}}, a_{0}=-\frac{\sigma}{\rho k}, a_{1}=0, b_{1}=-\frac{2 \delta k \mu}{\rho}
$$

Case 2:

$$
\mu=-\frac{\sigma^{2}}{8 \delta k^{3} a_{1} \rho}, \lambda=\frac{a_{1} \rho}{2 \delta k}, a_{0}=-\frac{\sigma}{\rho k}, a_{1}=a_{1}, b_{1}=\frac{\sigma^{2}}{4 k^{2} a_{1} \rho^{2}}
$$

Case 3:

$$
\mu=-\frac{\sigma^{2}}{4 \lambda k^{4} \delta^{2}}, \lambda=\lambda, a_{0}=-\frac{\sigma}{\rho k}, a_{1}=\frac{2 \delta k \lambda}{\rho}, b_{1}=0
$$

Substituting Eq. (36) with Eq. (13) into Eq. (35) and simplify, we obtain the following.

$$
U_{1}(\zeta)=-\frac{\sigma}{\rho k}+\frac{\sigma}{2 k \rho}\left[\frac{A \cosh \left(\frac{\sigma}{\delta k^{2}}\left(\frac{k x^{\alpha}}{\Gamma(1+\alpha)}+\frac{\sigma t^{\alpha}}{\Gamma(1+\alpha)}\right)\right)+A \sinh \left(\frac{\sigma}{\delta k^{2}}\left(\frac{k x^{\alpha}}{\Gamma(1+\alpha)}+\frac{\sigma t^{\alpha}}{\Gamma(1+\alpha)}\right)\right)+B}{A \cosh \left(\frac{\sigma}{\delta k^{2}}\left(\frac{k x^{\alpha}}{\Gamma(1+\alpha)}+\frac{\sigma t^{\alpha}}{\Gamma(1+\alpha)}\right)\right)+A \sinh \left(\frac{\sigma}{\delta k^{2}}\left(\frac{k x^{\alpha}}{\Gamma(1+\alpha)}+\frac{\sigma t^{\alpha}}{\Gamma(1+\alpha)}\right)\right)-B}\right]^{-1}
$$

Substituting Eq. (37) with Eq. (13) into Eq. (35) and simplify, we obtain the following.

$$
\begin{aligned}
U_{2}(\zeta) & =-\frac{\sigma}{\rho k}-\frac{\sigma}{2 k \rho}\left[\frac{A \cosh \left(\frac{\sigma}{2 \delta k^{2}}\left(\frac{k x^{\alpha}}{\Gamma(1+\alpha)}+\frac{\sigma t^{\alpha}}{\Gamma(1+\alpha)}\right)\right)+A \sinh \left(\frac{\sigma}{2 \delta k^{2}}\left(\frac{k x^{\alpha}}{\Gamma(1+\alpha)}+\frac{\sigma t^{\alpha}}{\Gamma(1+\alpha)}\right)\right)+B}{A \cosh \left(\frac{\sigma}{2 \delta k^{2}}\left(\frac{k x^{\alpha}}{\Gamma(1+\alpha)}+\frac{\sigma t^{\alpha}}{\Gamma(1+\alpha)}\right)\right)+A \sinh \left(\frac{\sigma}{2 \delta k^{2}}\left(\frac{k x^{\alpha}}{\Gamma(1+\alpha)}+\frac{\sigma t^{\alpha}}{\Gamma(1+\alpha)}\right)\right)-B}\right] \\
& -\frac{\sigma}{2 k \rho}\left[\frac{A \cosh \left(\frac{\sigma}{2 \delta k^{2}}\left(\frac{k x^{\alpha}}{\Gamma(1+\alpha)}+\frac{\sigma t^{\alpha}}{\Gamma(1+\alpha)}\right)\right)+A \sinh \left(\frac{\sigma}{2 \delta k^{2}}\left(\frac{k x^{\alpha}}{\Gamma(1+\alpha)}+\frac{\sigma t^{\alpha}}{\Gamma(1+\alpha)}\right)\right)+B}{A \cosh \left(\frac{\sigma}{2 \delta k^{2}}\left(\frac{k x^{\alpha}}{\Gamma(1+\alpha)}+\frac{\sigma t^{\alpha}}{\Gamma(1+\alpha)}\right)\right)+A \sinh \left(\frac{\sigma}{2 \delta k^{2}}\left(\frac{k x^{\alpha}}{\Gamma(1+\alpha)}+\frac{\sigma t^{\alpha}}{\Gamma(1+\alpha)}\right)\right)-B}\right]^{-1}
\end{aligned}
$$


Substituting Eq. (38) with Eq. (13) into Eq. (35) and simplify, we obtain the following.

$$
U_{3}(\zeta)=-\frac{\sigma}{\rho k}-\frac{\sigma}{\rho k}\left[\frac{A \cosh \left(\frac{\sigma}{\rho k^{2}}\left(\frac{k x^{\alpha}}{\Gamma(1+\alpha)}+\frac{\sigma t^{\alpha}}{\Gamma(1+\alpha)}\right)\right)+A \sinh \left(\frac{\sigma}{\rho k^{2}}\left(\frac{k x^{\alpha}}{\Gamma(1+\alpha)}+\frac{\sigma t^{\alpha}}{\Gamma(1+\alpha)}\right)\right)+B}{A \cosh \left(\frac{\sigma}{\rho k^{2}}\left(\frac{k x^{\alpha}}{\Gamma(1+\alpha)}+\frac{\sigma t^{\alpha}}{\Gamma(1+\alpha)}\right)\right)+A \sinh \left(\frac{\sigma}{\rho k^{2}}\left(\frac{k x^{\alpha}}{\Gamma(1+\alpha)}+\frac{\sigma t^{\alpha}}{\Gamma(1+\alpha)}\right)\right)-B}\right]
$$

Graphical representation of the solutions of Burgers equation: Presented Soliton periodic traveling wave solution $U_{1}(x, y)$ is surface wave solution (can occur along any boundary of two different substances) given in Figure 7. This figure gives the shape of the exact solution of fractional Burger equation.

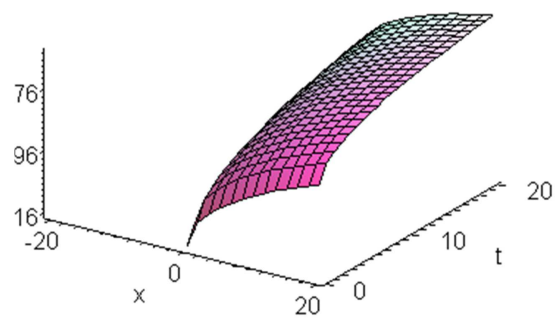

Figure 7. Modulus plot of kink wave shape of the solution $U_{1}(x, y)$ for $\rho=k=\sigma=1, A=3, B=6, \alpha=0.5, \delta=0.25$ with $0 \leq t \leq 20,-20 \leq x \leq 20$.

Presented Soliton periodic traveling wave solution $U_{2}(x, y)$ is singular kink wave solution given in Figure 8 . This figure gives the shape of the exact solution of fractional Burger equation.

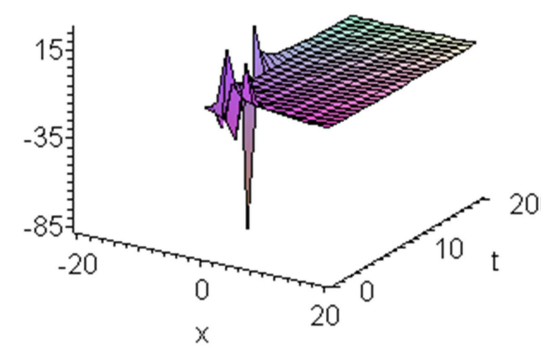

Figure 8. Exact for $\rho=k=\sigma=1, A=3, B=6, \alpha=0.5, \delta=0.25$ with $0 \leq t \leq 20,-20 \leq x \leq 20$.

Presented Soliton periodic traveling wave solution $U_{3}(x, y)$ is singular kink wave solution given in Figure 9. This figure gives the shape of the exact solution of fractional Burger equation.

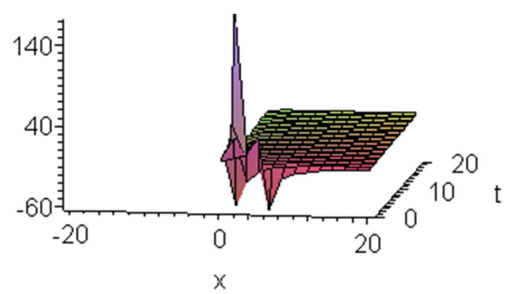

Figure 9. Exact for $\rho=k=\sigma=1, A=3, B=6, \alpha=0.5, \delta=0.25$ with $0 \leq t \leq 20,-20 \leq x \leq 20$.

\section{Conclusions}

In this paper, the $\left(G^{\prime} / G^{2}\right)$-expansion method has been applied to find some exact solutions of the two equations, fractional Burgers Fisher equation and fractional Burgers equation. Abundant exact traveling wave solutions are constructed for these equations by the proposed method. It is noteworthy to observe that our solutions are more general and contain further arbitrary constants and the arbitrary constants imply that these solutions have rich local structures. This method is effective and has a successful use in finding exact solutions of nonlinear fractional equations.

\section{References}

[1] A. Freihet, S. Hasan, M. Al-Smadi, M. Gaith, and S. Momani. (2019). Construction of fractional power series solutions to fractional stiff system using residual functions algorithm. Advances in difference equations, vol. 2019 (1), pp. 95.

[2] M. Alaroud, M. Al-Smadi, R. R. Ahmad, and U. K. Salma Din, (2019). An Analytical Numerical Method for Solving Fuzzy Fractional Volterra Integro-Differential Equations. Symmetry, vol. 11 (2), pp. 205.

[3] Saleh Alshammari, Mohammed Al-Smadi, Mohammad Al Shammari, Ishak Hashim, and Mohd Almie Alias, (2019). Advanced Analytical Treatment of Fractional Logistic Equations Based on Residual Error Functions. International journal of differential equations, vol. 2019, pp. 1-11.

[4] S. Hasan, M. Al-Smadi, A. Freihet, and S. Momani, (2019). Two computational approaches for solving a fractional obstacle system in Hilbert space. Springer International publishing AG, vol. 2019 (1), pp. 55.

[5] Cheng Chen and Yao-Lin Jiang, (2018). Simplest equation method for some time-fractional partial differential equations with conformable derivative. Computers and Mathematics with Applications, vol. 75, Issue 8, pp. 2978-2988.

[6] E. A. Yousif, E. A-B. Abdel-Salam, M. A. El-Aasser, (2018). On the solution of the space-time fractional cubic nonlinear Schrödinger equation. Elsevier, Results in Physics, vol. 8, pp. 702-708.

[7] M. Alaroud, M. Al-Smadi, R. R. Ahmad, and U. K. Salma Din, (2018). Computational optimization of residual power series algorithm for certain classes of fuzzy fractional differential equations. International journal of differential Equations, vol. 2018, pp. 1-11.

[8] M. Ali Akbar, Norhashidah Hj. Mohd. Ali, Ripan Roy, (2018). Closed form solutions of two time fractional nonlinear wave equations. Elsevier, Results in Physics, vol. 9, pp. 1031-1039. 
[9] Shumaila Javeeda, Summaya Saifa, Asif Waheedb and Dumitru Baleanuc, (2018) Exact solutions of fractional mBBM equation and coupled system of fractional BoussinesqBurgers. Elsevier, Results in Physics, vol. 9, pp. 1275-1281.

[10] Arzu Akbulut a and Melike Kaplan, (2018). Auxiliary equation method for time-fractional differential equations with conformable derivative. Computers and Mathematics with Applications, vol. 75, Issue 3, pp. 876-882.

[11] Dazhi Zhao. Maokang Luo (2017). General conformable fractional derivative and its physical interpretation. SpringerVerlag Italia, 54, pp. 903-917.

[12] K. Hosseini and R. Ansari,(2017). New exact solutions of nonlinear conformable time-fractional Boussinesq equations using the modified Kudryashov method, Waves in Random and Complex Media, vol. 27, pp. 628-636.
[13] Nematollah Kadkhoda (2017). Application of $\left(G / G^{2}\right)$ expansion method for solving fractional differential equations. International journal of applied and computational mathematics, 3, pp. 1415-1424.

[14] I. Hashim, (2016). Solutions to Uncertain Volterra Integral Equations by Fitted Reproducing Kernel Hilbert Space Method. Journal of Function Spaces, vol. 2016, pp. 1-11.

[15] A. H. Arnous, (2015). Solitary wave solutions of space-time FDEs using the generalized Kudryashov method. Acta universitatis apulensis, 42, pp. 41-51.

[16] Thabet Abdeljawad, (2015). On conformable fractional On conformable fractional calculus. Journal of computational and applied mathematics, Vol. 279, pp. 57-66. 